\title{
A Fiber Optic Probe for Monitoring Protein Aggregation, Nucleation, and Crystallization
}

Rafat R. Ansari and Kwang I. Suh

Lewis Research Center

Cleveland, Ohio

Alireza Arabshahi and W. William Wilson

Mississippi State University

Mississippi State University, Mississippi

Terry L. Bray and Lawrence J. DeLucas

University of Alabama at Birmingham

Birmingham, Alabama

Prepared for the

Sixth International Conference on Crystallization of Biological Macromolecules sponsored by the Hiroshima Prefecture, Hiroshima City, the Ministry of Education, Science, Sports and Culture, and the International Union of Crystallography Hiroshima, Japan, November 12-17, 1995

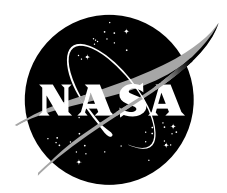

National Aeronautics and

Space Administration 


\title{
A FIBER OPTIC PROBE FOR MONITORING PROTEIN AGGREGATION, NUCLEATION, AND CRYSTALLIZATION
}

\author{
R.R. Ansari and K.I. Suh \\ National Aeronautics and Space Administration \\ Lewis Research Center \\ Cleveland, Ohio 44135 \\ A. Arabshahi and W.W. Wilson \\ Mississippi State University \\ Mississippi State, Mississippi 39762 \\ and \\ T.L. Bray and L.J. DeLucas \\ The University of Alabama \\ Birmingham, Alabama 35294
}

\begin{abstract}
SUMMARY
Protein crystals are often experimentally grown in hanging drops in microgravity experiments on-board the Space Shuttle orbiter. The technique of dynamic light scattering (DLS) can be used to monitor crystal growth processes in hanging droplets $(\sim 30 \mu \mathrm{L})$ in microgravity experiments, but elaborate instrumentation and optical alignment problems have made in-situ applications difficult. In this paper we demonstrate that such experiments are now feasible. We apply a newly developed fiber optic probe to various earth and space (microgravity) protein crystallization system configurations to test its capabilities. These include conventional batch (cuvette or capillary) systems, a hanging drop method in a six-pack hanging drop vapor diffusion apparatus (HDVDA), a modified HDVDA for temperature-induced nucleation and aggregation studies, and a newly envisioned dynamically controlled vapor diffusion system (DCVDS) configuration. Our compact system exploits the principles of DLS and offers a fast (within a few seconds) means of quantitatively and noninvasively monitoring the various growth stages of protein crystallization. In addition to DLS capability, the probe can also be used for performing single-angle static light scattering measurements. It utilizes extremely low levels of laser power ( a few $\mu \mathrm{W}$ ) and essentially eliminates the usual problems associated with optical alignment and vibration isolation. The compact probe is also equipped with a miniaturized microscope for visualization of macroscopic protein crystals. This new optical diagnostic system makes possible the exploration of new ways to grow good quality crystals suitable for $\mathrm{x}$-ray crystallographic analysis and may contribute to a concrete scientific basis for understanding the process of crystallization.
\end{abstract}

\section{INTRODUCTION}

The growth of protein crystals is usually the rate limiting step in the determination of three dimensional structures of biologically important proteins. This area has been of particular interest in recent years due to the use of structure based rational drug design for developing new pharmaceutical materials. This approach relies on the determination of the three dimensional structure of a specific protein related to a particular disease process. Once the structure has been determined and the appropriate active site(s) of the protein have been identified, then a drug can be designed to modify the protein's activity and interrupt the disease process. The only direct method for determining three dimensional structures of large biological proteins is the technique of $\mathrm{x}$-ray crystallography which requires well ordered crystals of the target protein in order to solve the structure. Obtaining these crystals is typically the most difficult step prior to using x-ray crystallography. This difficulty led to the exploration of other avenues which might yield high quality crystals. Early experiments by Walter Littke (ref. 1) led researchers in the U.S. to investigate the effects of eliminating gravity on crystal growth. This interest ultimately resulted in the development of a hanging drop vapor diffusion apparatus (HDVDA). This hardware, described by Rosenblum et al. (ref. 2), has been 
used on Space Shuttle missions to successfully grow crystals of several proteins which have produced x-ray data surpassing the data collected from the best earth grown crystals. However, not all proteins have produced better crystals in space. This is believed to occur because the conditions for crystal growth are different in a microgravity environment than in $1 \mathrm{~g}$.

The fundamental physics for crystallization of biological macromolecules is still not well understood. Small molecules (e.g., $\mathrm{NaCl}$ ) have stronger bonds holding the crystal together than do protein crystals which are typically held together by much weaker electrostatic interactions, hydrogen bonds, and water bridges. Protein crystals also contain a great deal of solvent, typically anywhere from 40 to 80 percent of the crystal volume. These factors make protein crystals much more difficult to crystallize in a well ordered manner suitable for x-ray diffraction studies. While there are several theories regarding the mechanism of protein crystallization, it is not definitively clear whether monomers or some higher order aggregate(s) comprise the growth unit. There is evidence supporting both theories. It is possible both mechanisms contribute to crystal growth simultaneously. A concrete physical understanding of the protein crystallization process at a fundamental science level would require detailed studies of the nucleation and post-nucleation growth phenomena. Since nucleation may depend upon temperature, monomer concentration, $\mathrm{pH}$, solution ionic strength and crystallizing agent concentration, dynamic control of one or more of these parameters and a specific monitoring technique would be very useful. Dynamic control of nucleation offers the possibility of a more systematic approach to protein crystal growth, and its implementation calls for the development of suitable quantitative and noninvasive monitoring techniques.

Since the evaporation rate in a HDVDA experiment is set by the initial solution concentrations, active control over the equilibration rate of the growth solution is not possible once the experiment is activated. This limitation has led to the development of a dynamically controlled vapor diffusion system (DCVDS), described by Bray et al. (ref. 3), which does allow active control over evaporation rates. The DCVDS utilizes humidity sensors and a laser scattering detector which allow control over the approach to nucleation, detection of nucleation and control of postnucleation growth. The major goal of this study is to determine if the optical probe described in this paper provides the necessary features as a laser scattering diagnostic so that it meets the requirements for the DCVDS and other ground based and space based systems. The preliminary results indicate that the probe does meet this objective.

\section{Data Analysis}

The technique of DLS is commonly used in the characterization of macromolecular solutions and its principles are documented in several books such as the one by $\mathrm{Chu}$ (ref. 4). The incident laser radiation interacts with the particles dispersed in a fluid. The particles scatter light and the intensity of this scattered light fluctuates in time due to the Brownian or thermal motion of the dispersed particles in the suspending medium. When a dynamic medium such as a protein solution is illuminated by laser light, a rapidly changing interference pattern due to the differences in the optical density of the scattering medium is produced and contains information about the dynamics of the scattering medium which can be extracted by constructing a time autocorrelation function (TCF) (refs. 4 and 5). The TCF is computed using a digital correlator, and in the case of dilute dispersions of spherical particles, the TCF provides quick and accurate determination of the particle translation diffusion coefficient. The diffusion coefficient data can easily be transformed into average particle size, using the Stokes-Einstein equation, provided the viscosity of the suspending medium, its temperature and refractive index are known.

Conventionally the technique of DLS has been used for studying dilute solution properties of spherically shaped particles of effective size ranging between $3 \mathrm{~nm}$ and $3 \mu \mathrm{m}$. For concentrated suspensions exhibiting multiple light scattering effects and for dispersions containing more than one scattering species, however, the data analysis and interpretation of the TCF becomes more difficult. The protein systems studied in this paper do not exhibit multiple light scattering effects. Our DLS probe is capable of handling concentrated dispersions. Ansari et al. (ref. 6) have accurately determined, without a need to correct for multiple light scattering, the alpha crystalline protein size in the eye lens where its composition is very high ( 35 to 40 percent $w / v$ ). We must note, however, that the TCF data obtained during post-nucleation protein crystal growth where nonspherical micron sized particles are present must be analyzed and interpreted with extreme care. When particle size approaches $\sim 1 \mu \mathrm{m}$ in ground-based systems, sedimentation often becomes dominant. Therefore, DLS data should only be used to detect gross trends when particle sizes become larger and the assignment of specific size values in absolute terms is qualitative. The TCF data presented here was analyzed using the commercial data inversion routines supplied by the Brookhaven Instruments 
Corporation (Holtsville, NY). These include a method of cumulants, double exponential, and a computer algorithm called CONTIN. These data analysis techniques and their comparison are described in detail by Stock and Ray (ref. 7).

\section{Optical Design}

We chose to exploit the technique of DLS to reliably characterize dynamical properties (diffusion coefficients or size) of the protein molecules in a hanging drop. Until recently such experiments were not possible because of optical alignment problems, control of penetration depth inside the sample, physical size of the instrumentation and power requirements for the laser and photodetectors. In the last few years, however, the DLS instrumentation has profited from technologies associated with the advent of miniaturization in solid state lasers, photodetectors, optical components and fiber optic communication devices (ref. 8).

One of the difficulties in probing protein monomers in dilute solutions is their small size ( 3 to $8 \mathrm{~nm}$ in diam). Conventional DLS systems require laser powers of the order of a few hundred milliwatts and data collection times of minutes to obtain a good-quality TCF. One factor affecting the TCF, as shown by Ford (ref. 5), is the precise control of the scattering volume or coherence area in the sample. In conventional DLS systems the coherence area is controlled by making use of lenses and apertures as part of the launch and detection optics. Fiber optic systems reported by Dhadwal (ref. 9) have made use of two optical fibers, one for launch and the other for detection, mounted on one gradient index (GRIN) microlens. A lensless fiber optic system for doing DLS measurements has also been reported by Dhadwal et al. (ref. 10). The former (fig. 1 and 1(a) in ref. 9) can only be used as an immersion probe while the lensless probe (ref. 10) can be used both inside and outside the sample cell. Both designs have certain limitations. In the former case, in our experience, reflections arising from the lens and fiber surfaces give rise to heterodyning effects thus compromising the reliability and accuracy in obtaining correct particle sizes. The lensless fiber optic system, on the other hand, solves the problem of spurious reflection, but for outside use it must be placed within a very close proximity $(\leq 2 \mathrm{~mm})$ of the sample being interrogated. Further, its use is limited in interrogating very dilute samples when used in conjunction with solid-state lasers and samples of larger colloidal particles. The sensitivity and reliability are drastically reduced for particles greater than 300 to $500 \mathrm{~nm}$ because of extremely low spatial coherence $(\beta)$ values and a large scattering area $\left(\sim 5 \mathrm{~mm}^{2}\right)$. This system is therefore not suitable for the HDVDA or DCVDS system configurations.

Casay and Wilson (ref. 11) have successfully demonstrated the use of static and DLS techniques in the only reported study of the lysozyme nucleation process in a modified HDVDA. In that study separate laser launch and detection systems were used. The laser launch system employed a multimode optical fiber with a microlens to produce a collimated beam of light in the sample. The scattered light was collected at a scattering angle of $90^{\circ}$ using a single mode optical fiber. The separate laser and detector systems compromise flexibility and demand a high degree of optical alignment which is not easy to achieve in a microgravity environment. Although the experimental results were good, their system was not optimized for the most efficient self-beating (DLS) light scattering experiments. Further, such a system cannot be used on a DCVDS system which employs sample cells fabricated out of acrylic material which becomes opaque upon machining.

Our newly designed system alleviates all of the above problems. We attach separate micro-graded index (GRIN) lenses on each optical fiber (launch and detection) in such a way that the scattering volume $\left(\sim 2 \times 10^{-5} \mathrm{~mm}^{3}\right)$ and coherence area (ref. 5) are very tightly controlled resulting in high spatial coherence $(\beta>0.5)$ values. A fiber optic probe comprised of two monomode optical fibers and two GRIN microlenses, as illustrated in figure 1, provides a compact and remote means of studying the dynamical characteristics of the protein particles in a hanging droplet. The fiber optic probe $(1.3 \mathrm{~cm}$ in diam $\times 1.3 \mathrm{~cm}$ in length) contains the necessary optics to perform DLS measurements at a scattering angle of $161.5^{\circ}$. The probe is shown in figure 1 with a U.S. dime for size comparison. The probe is noninvasive and is conveniently positioned in front of the drop, but has no physical contact with any part of the sample or its container. Two monomode optical fibers, each housed in a stainless steel ferrule, are mounted into a separate stainless steel housing. An air gap ( 0 to $0.05 \mathrm{~mm}$ ) is intentionally left between the fiber housing and the lens housing in order to produce a tightly focused spot in the scattering volume. The two optical fibers in their housings are aligned and fixed into position off-axis with the GRIN lens. The two housings are placed inside a third (outer) housing made of stainless steel, and the back end of the housing is covered with a heat-shrink tubing. The two free ends of the optical fibers were terminated with FC/PC-type male connectors for easy mating with the laser/detectormodule comprised of a temperature-controlled laser diode (Toshiba model TOLD 9215), a 
constant power driver, a photomultiplier tube (PMT) (Hamamatsu HC-120) and an amplifier-discriminator system (Brookhaven Instruments Corporation, NY). An avalanche photo diode (APD) photon counting module (model PCS-2, EG and G Canada) was also used as a photodetector. The PMT and the APD were used to collect scattered light from concentrated polystyrene solutions and protein solutions, respectively.

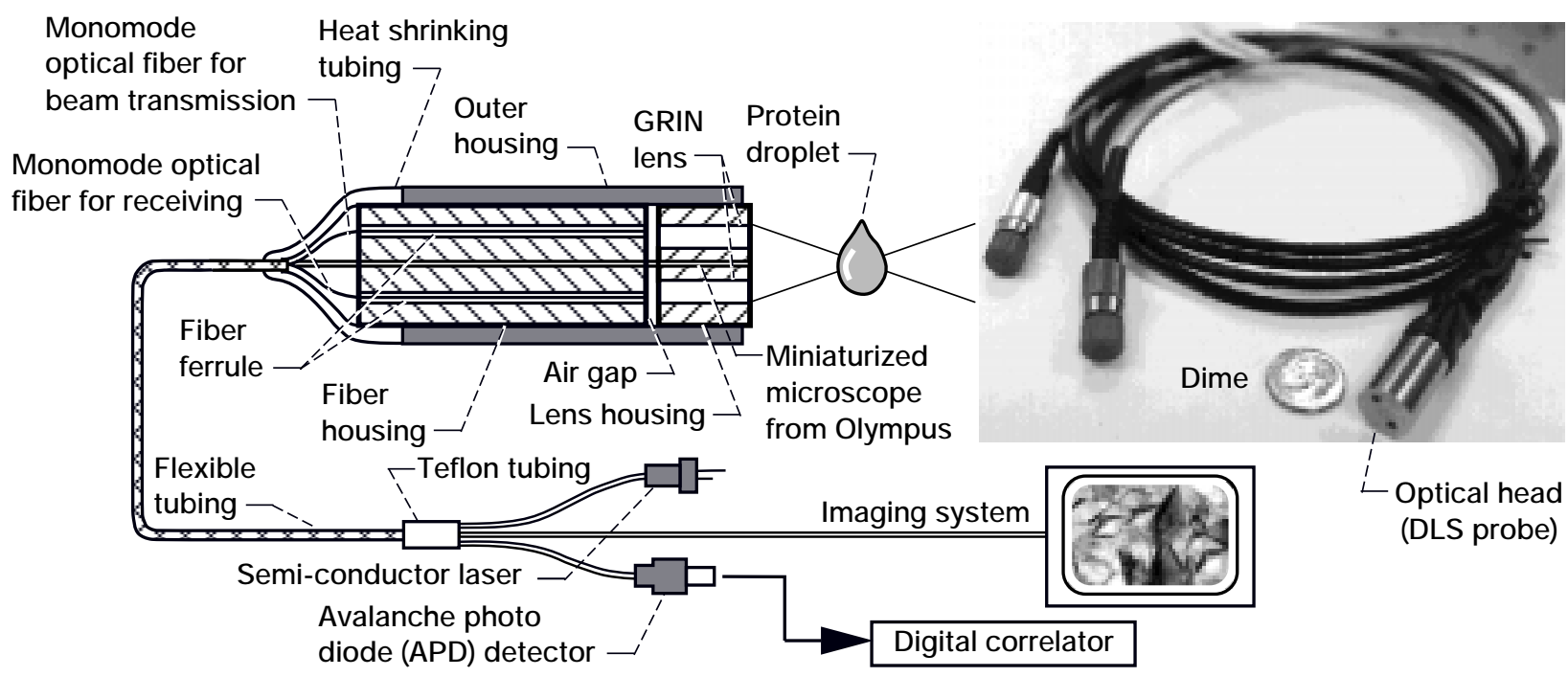

Figure 1.-A compact optical probe for monitoring protein crystallization.

Our optical design (probe) provides major improvements over previously reported DLS systems. These improvements include accurate determination of particle sizes from very small $(3 \mathrm{~nm})$ to very large $(\sim 1 \mu \mathrm{m})$ with very short data acquisition times (within seconds) and extremely small laser power levels (few $\mu \mathrm{W}$ ) from a laser diode, and the ability to place the probe outside the sample container anywhere from 0 to $17 \mathrm{~mm}$ from the sample's surface. The probe can also be immersed in the solution. Another major improvement is its ability to probe solution dynamics in sample containers of varying size and shapes. We have performed accurate and reliable particle size measurements with this probe on dispersions contained in regular spectrographic cuvettes, capillary tubes, common laboratory utensils (beakers, graduated cylinders, conical flasks, glass/clear plastic container vessels), concentric cells containing two different solutions and hanging fluid drops. Extremely small sample quantities of protein solutions $(\sim 30 \mu \mathrm{L})$ were interrogated in the experiments reported in this paper. Ansari and Suh (ref. 12) have recently shown that the performance of their probe is far better when compared with the lensless probe of Dhadwal et al.

(ref. 9). The newly designed fiber optic probe was thoroughly tested using polystyrene monodisperse standards ranging in size from $20 \mathrm{~nm}$ to $0.8 \mu \mathrm{m}$ at several concentrations and bovine serum albumin (BSA) protein samples ranging in concentration from 2 to 10 percent $(\mathrm{w} / \mathrm{v})$. These measurements were performed in batch mode and in hanging drops and were recently reported by Ansari and Suh (ref. 12). The effect of data acquisition time (experiment duration) and the incident laser power using the probe was also tested with regard to yielding correct particle sizes. The results showed that accurate particle size measurements were obtainable in $5 \mathrm{~s}$ at a laser power of $0.2 \mathrm{~mW}$. Such short measurement times may be useful for studying nucleation and growth phenomena. Recently, Ansari et al. (ref. 6) have used this probe to detect early signs of eye diseases (e.g., cataracts) in live animals and cadavers.

\section{Experimental Set Up and Procedure}

The experimental set up was comprised of four parts; a temperature-controlled laser/detector module, a protein crystal growth facility (cuvette, HDVDA and DCVDS), a fiber optic probe and a data acquisition system. The experimental configurations are shown in figures 2 and 3. The probe was mounted on the HDVDA/DCVDS using a V-clamp and an optical post. Laser light $(\lambda=670 \mathrm{~nm})$ from a laser diode at $80 \mu \mathrm{W}$ power level was launched into the sample via the probe. Light scattered from the particles inside the scattering volume $\left(\sim 2 \times 10^{-5} \mathrm{~mm}^{3}\right)$ was collected at a scattering angle of $161.5^{\circ}$ by the same probe assembly and was guided to the PMT or APD. The resulting signal 


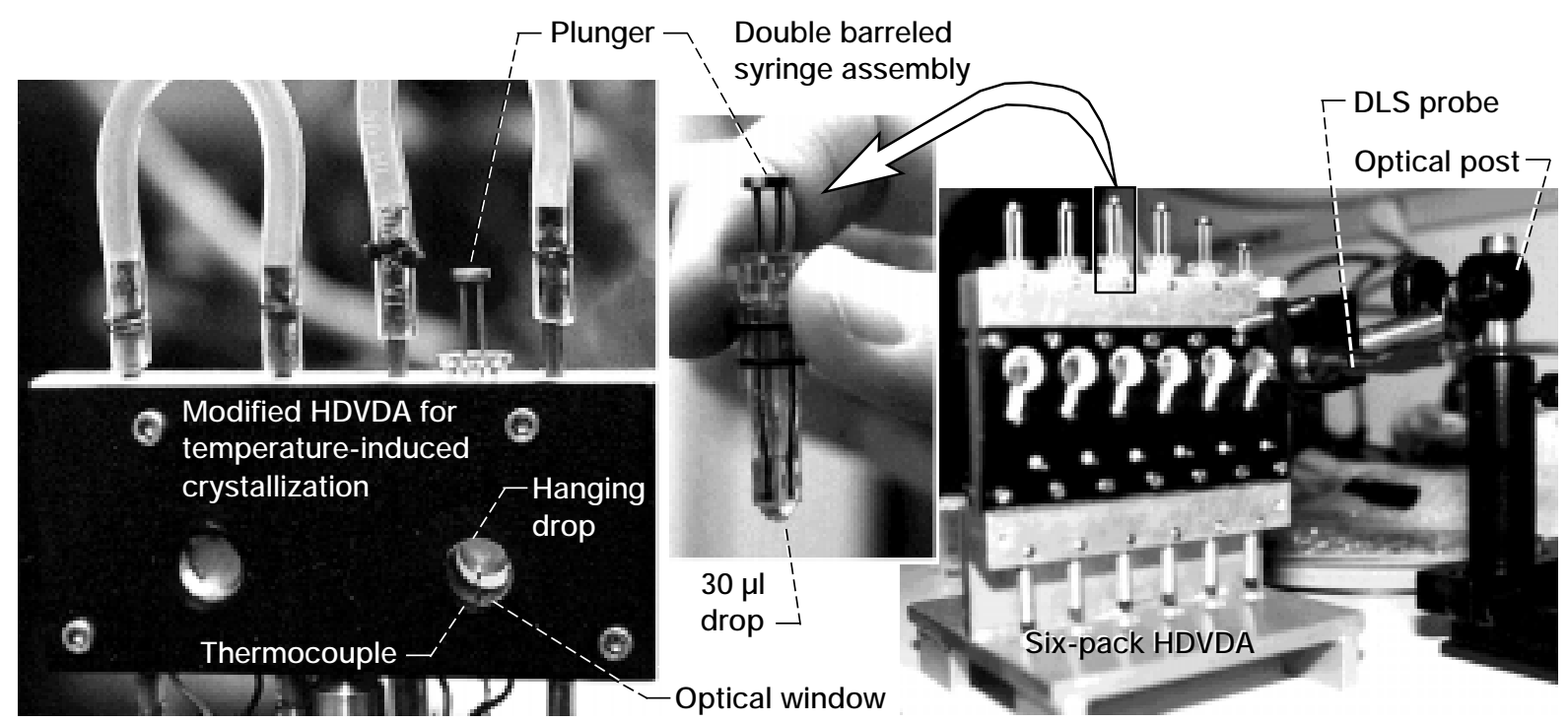

Figure 2.-Experimental setup: hanging drop vapor diffusion apparatus (HDVDA) configuration.

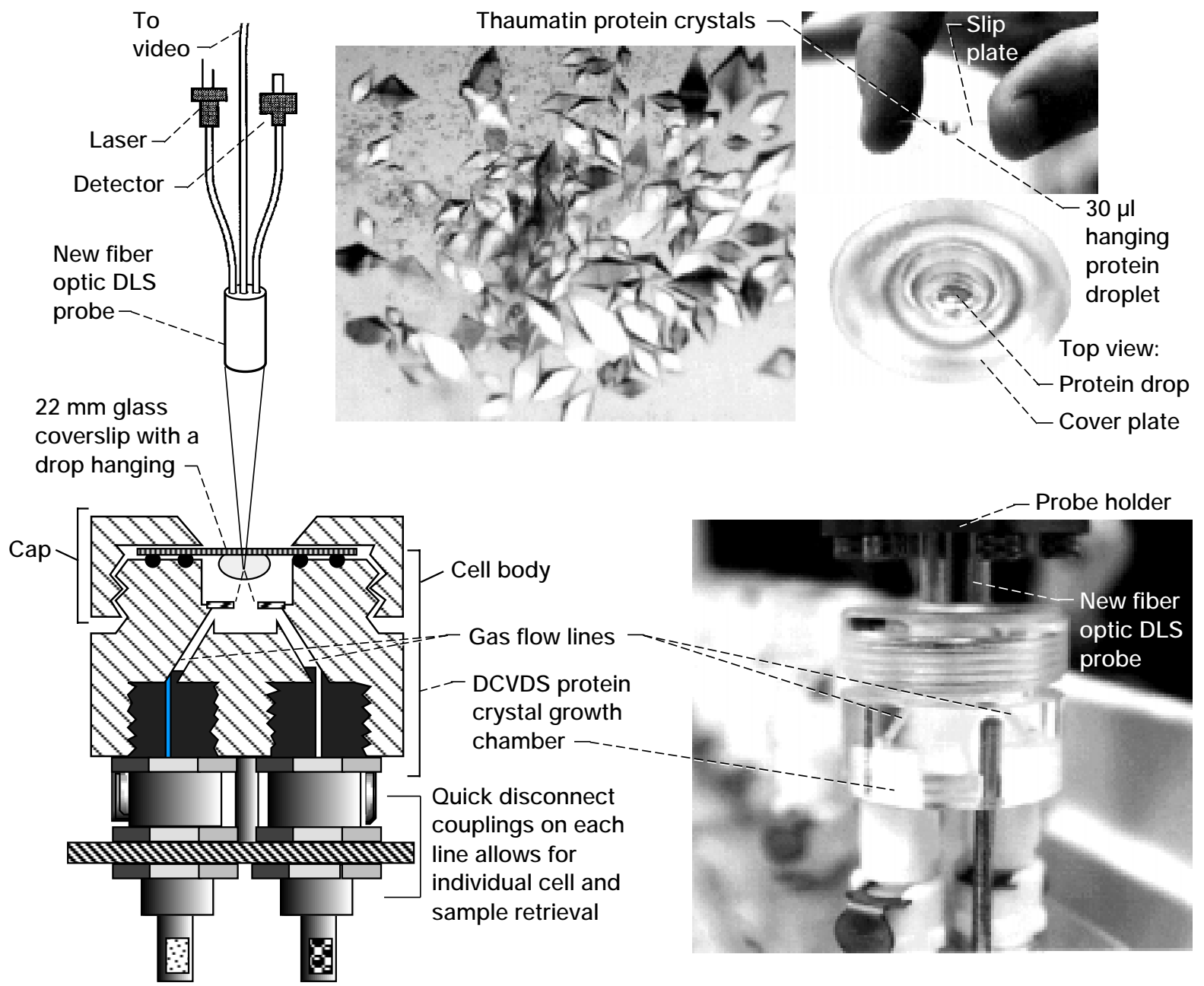

Figure 3.-Experimental setup: dynamic control vapor diffusion system (DCVDS) configuration. 
was fed to a digital correlator card (Brookhaven Instruments BI9000) housed in a docking station for a Pentium ${ }^{\mathrm{TM}}$ based notebook computer (7600 Series, EPS). A logarithmic correlation time scaling was used to capture the dynamics of very small and very large particles which may be present in the same scattering volume. Another unique feature of our DLS probe is that it is equipped with a miniaturized microscope which can be used for visualizing macroscopic particles once they appear during a crystallization experiment. However, in the experiments reported in this paper this feature was not utilized in on-line configuration because of inadequate white-light sample illumination conditions in the experimental chambers.

The first apparatus tested was a modified HDVDA. This apparatus, shown in figure 2, is a modification of space flight hardware which mimics the hanging drop vapor diffusion method for growing protein crystals. The HDVDA is equipped with an optical window made of plexiglass. This window has a thickness of $3.8 \mathrm{~mm}$ and the distance from the front of the hanging droplet to the back surface of the window is $2.5 \mathrm{~mm}$. In the HDVDA, the growth solution is suspended from a double barreled syringe which is surrounded by a wicking material containing the reservoir solution. Initial solution concentrations are chosen such that water diffuses from the droplet into the reservoir. In the modified HDVDA, the wicking material was removed and the droplet chamber size was reduced (fig. 2). Thermoelectric devices were embedded around the chamber so that temperature induced crystallization could be performed instead of vapor diffusion. The protein solution was placed in one side of the double barreled syringe and the crystallizing agent in the other side. The syringe was then placed in the HDVDA and the two solutions were deployed together as a droplet.

The second apparatus used was a dynamically controlled vapor diffusion system (DCVDS), shown in figure 3, which also mimics the hanging drop vapor diffusion method. This device, however, uses controlled sequential purges of a dry nitrogen gas to evaporate water from the growth solution at a user prescribed rate. This approach replaces the reservoir solution used in the traditional HDVDA for accomplishing vapor diffusion. A humidity sensor is used to monitor the amount of water evaporated out of the growth solution as a function of time. The feedback from this sensor can be used to effect virtually any evaporation profile. A microcomputer utilizing custom software and data acquisition/interface boards operates and monitors all aspects of the system.

\section{Sample Preparation}

A $40 \mathrm{mM}$ sodium acetate buffer solution was prepared at $\mathrm{pH} 4.3$ and was used to dissolve lysozyme from Boehringer to a concentration of $38.2 \mathrm{mg} / \mathrm{ml}$. The same buffer was used to prepare a 7 percent $(\mathrm{w} / \mathrm{v}) \mathrm{NaCl}$ stock solution used as a crystallizing agent. Thaumatin from sigma was dissolved in 50mM ADA buffer at pH 6.5 to a concentration of $68.5 \mathrm{mg} / \mathrm{ml}$. The same buffer was used to prepare a stock solution of NaK tartrate at $0.075 \mathrm{M}$. All solutions were filtered through $0.2 \mu \mathrm{m}$ Acrodisc filters.

\section{RESULTS AND DISCUSSION}

Detection of the Onset of Temperature-Induced Aggregation in a Modified HDVDA.-We first tested the feasibility of our probe to detect the onset of temperature-induced aggregation in a $25 \mathrm{mg} / \mathrm{ml}$ solution of lysozyme dissolved in $40 \mathrm{mM}$ acetate buffer, $\mathrm{pH}=4.3$, with 2.5 percent $\mathrm{w} / \mathrm{v} \mathrm{NaCl}$. These experiments were conducted in a modified HDVDA as shown in figure 2. The average particle size and the average scattered intensity (countrate) were monitored simultaneously as a function of time as shown in figure 4(a). The method of cumulants was used to reduce the DLS data to particle size. We distinguish three distinct regions in this plot. The experiment temperature began at $27^{\circ} \mathrm{C}$ and was kept constant for $1 \mathrm{hr}$. The data was collected every $60 \mathrm{sec}$ for a duration of $30 \mathrm{sec}$. At stage 1 (up to $1 \mathrm{hr}$ ) the average scatted intensity and average size of the protein molecules remained essentially constant (intensity within 1.34 percent and size within 2 percent). After this time the solution temperature was ramped down to $16^{\circ} \mathrm{C}$ at a rate of $1{ }^{\circ} \mathrm{C} / \mathrm{min}$. At stage 2 both the average scattered intensity and average particle size increased, and we interpret this as an indication of the onset of protein aggregation. This behavior corroborates the earlier observations of Casay and Wilson (ref. 11) on the basis of their DLS and static light scattering measurements. The temperature was then ramped up to $21^{\circ} \mathrm{C}$ at $1{ }^{\circ} \mathrm{C} / \mathrm{min}$ and maintained until the total experiment time was about $3 \mathrm{hr}$. At stage 3 we note a reduction in average size of the particles and average scattered intensity indicating some degree of dissociation of the primary aggregates. Data selected from the three stages was analyzed using CONTIN and is plotted in figure 4(b). The histogram presentation shows the shifts in average particle size corresponding to 

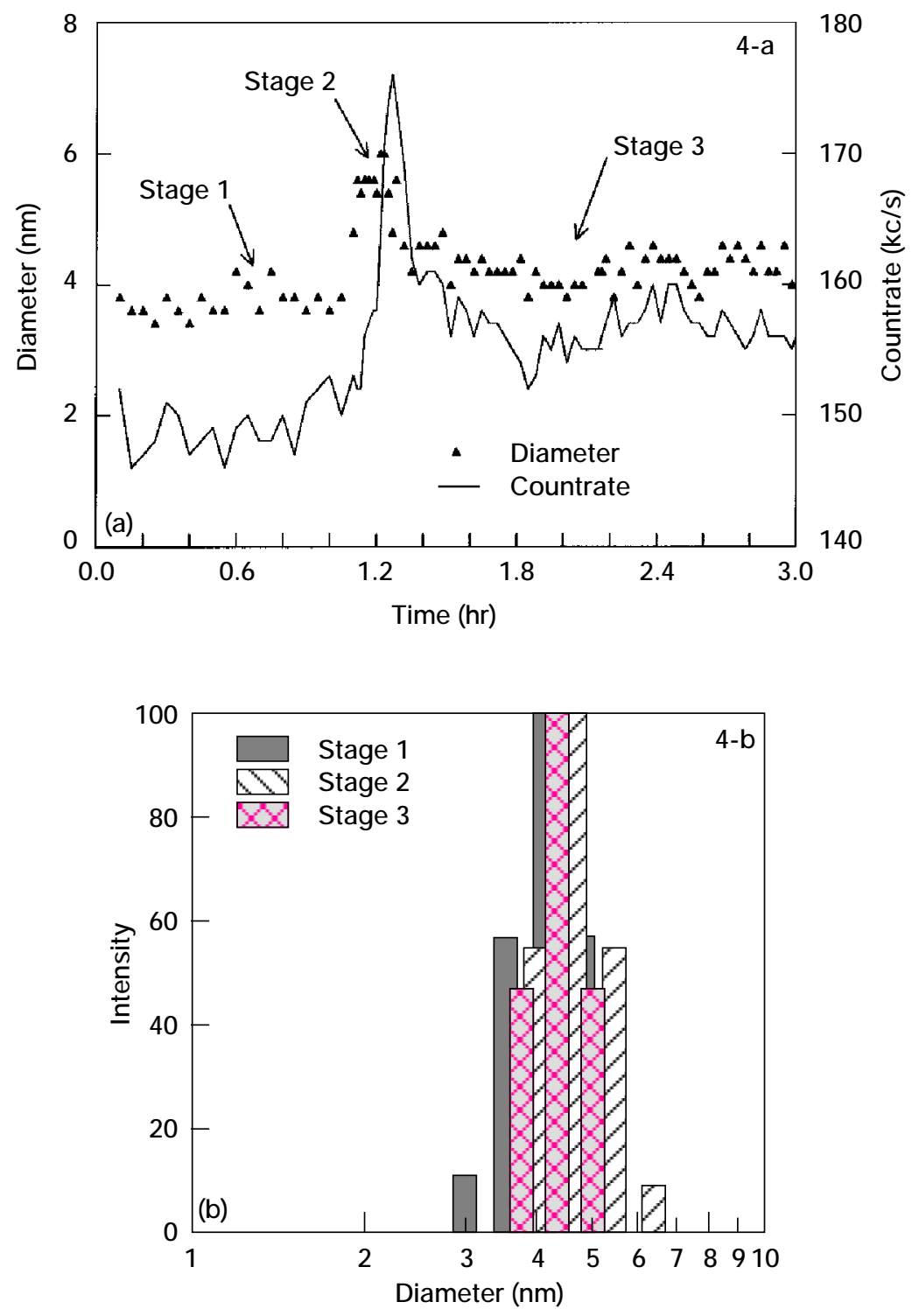

Figure 4.-Detection of the onset of temperature-induced aggregation in a hanging drop of lysozyme solution. (a) Average particle size and average scattered intensity as a function of time. (b) Size distribution.

stages 1 to 3 and is consistent with the cumulant analysis data shown in figure 4(a). A polydispersity factor, described in reference 4 and measured from the DLS-TCF data, can be a sensitive measure of aggregation. In our experiments, this factor, often referred to as the poly, increased from 0.005 in the initial stage to 0.22 in the later state suggesting a change from a nearly monodisperse state to a more polydisperse state. The state of protein aggregation corresponding to stage 1 is clearly best described as that of lysozyme monomer since the average particle diameter was determined to be about $3.8 \mathrm{~nm}$ and the poly value was extremely low. At stage 2, the average diameter increased to between 5 and $6 \mathrm{~nm}$ with an accompanying increase in poly. The actual particle size distribution at stage 2 is indeterminate from these data, and no inferences concerning specific monometer $\leftrightarrow n$-mer equilibria are made. However, from these results we do conclude that our newly designed probe is quite sensitive for detection of the aggregation stages in a dynamically controlled protein solution environment.

Detection of Fast Crystallization in the HDVDA and DCVDS.- The results described in the previous section (section 5.1) were for lysozyme solutions at relatively low supersaturation. The data analysis showed that the aggregates detected were small with no indication of particle sizes greater than $10 \mathrm{~nm}$ and certainly nothing of microscopic 
size. But what about the case when high protein supersaturations are employed? Crystallization in some proteins can be induced relatively quickly with large populations of microcrystals formed. In order to test the latter case, two experiments were performed utilizing supersaturated lysozyme in the HDVDA and supersaturated thaumatin in the DCVDS. The first experiment tested the probe on a supersaturated solution of $85 \mathrm{mg} / \mathrm{ml}$ lysozyme in $40 \mathrm{mM}$ acetate buffer, $\mathrm{pH}=4.3$ with 4 percent $\mathrm{NaCl}$. The lysozyme solution was loaded in the six-pack HDVDA unit at an initial temperature of $24^{\circ} \mathrm{C}$. The average particle size and average scattered intensity as a function of time is plotted in figure 5. The initial particle sizes in this solution are dramatically different from that depicted in figure 4(a). This solution clearly contained particles that had an average diameter of several hundred $\mathrm{nm}$, not surprising for such a highly supersaturated solution. The temperature, $24^{\circ} \mathrm{C}$, was held constant for about 40 min during which the average particle size was maintained. After about $40 \mathrm{~min}$ the temperature of the lysozyme solution was quickly reduced to $21{ }^{\circ} \mathrm{C}$. Immediately upon cooling, both average size and average scattered intensity increased and finally, lysozyme crystals ( $\sim 0.1$ to $0.2 \mathrm{~mm}$ ) appeared. This general trend in the increase in particle size has been noted by Malkin and McPherson (ref. 13) in a light scattering study of STMV, apoferritin and pumpkin globulin at several different high supersaturation levels. Figure 6(a) shows typical TCF profiles at the initial, intermediate and crystallizing stages of the solution. The first TCF $(t=0)$, an intermediate TCF $(t=40 \mathrm{~min})$ and last TCF $(\mathrm{t}=55 \mathrm{~min})$ show significant change in their shape. This is reflected in the histogram presentations of particle size shown in figure 6(b). The initial particles $(\mathrm{t}=0)$ had sizes centered around $5 \mathrm{~nm}$ and $0.5 \mu \mathrm{m}$ while the particles at the crystallizing stage $(\mathrm{t}=55 \mathrm{~min})$ showed two sizes; one centered around $8 \mathrm{~nm}$ and another $5 \mu \mathrm{m}$. These measurements indicate the sensitivity of the DLS probe for detecting the presence of both small and large particles in the same solution. We do not wish to emphasize the absolute values for the particle size distributions, but instead, point out the good dynamic range of the DLS probe.

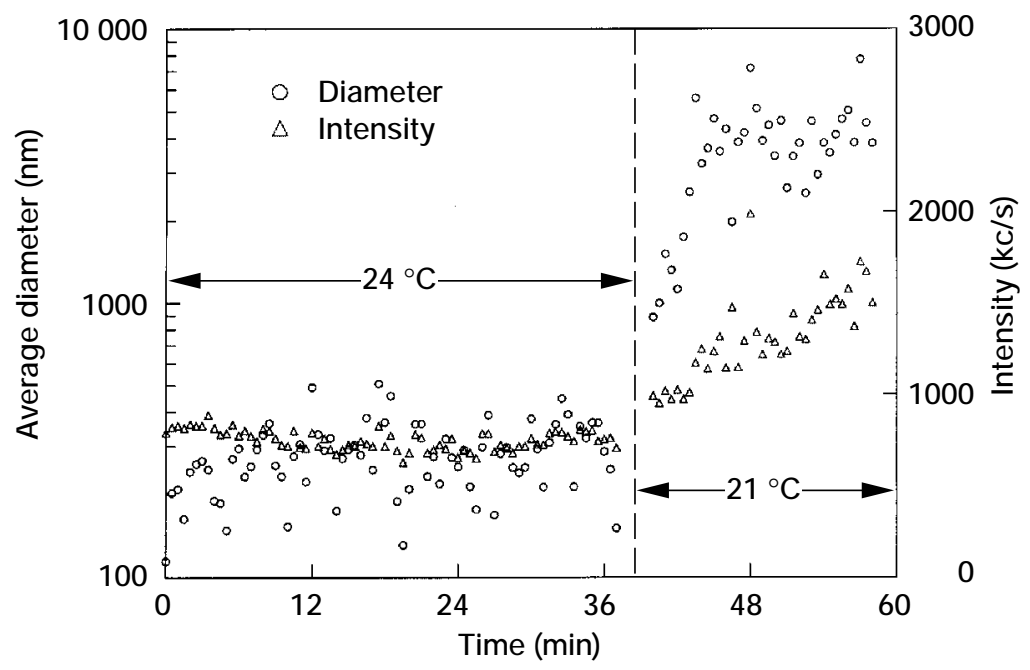

Figure 5.-Temperature-induced crystallization in a lysozyme system in a six-pack HDVDA.

DLS measurements were also performed on another supersaturated solution comprised of $34.35 \mathrm{mg} / \mathrm{ml}$ thaumatin mixed with $0.038 \mathrm{M} \mathrm{NaK}$ tartrate in the DCVDS at the highest rate of evaporation. The TCF profiles are plotted in figure 7. The $\mathrm{t}=0$ measurement shows a particle size centered around $5 \mathrm{~nm}$ which can be regarded as thaumatin monomers. After 4 min of evaporation the size distribution increases very rapidly centering around $4.3 \mathrm{~nm}$ and $13 \mu \mathrm{m}$. Ten minutes later the solution was examined again. It showed a size distribution centered around $4.7 \mathrm{~nm}$ suggesting sedimentation of large crystals. Indeed upon examination under a microscope the crystals ( 0.1 to $0.2 \mathrm{~mm})$ appeared at the bottom of the hanging thaumatin droplet and are shown in figure 3. Again, we have demonstrated the effectiveness of the DLS probe for detecting large changes in particle size that can be forced to occur in a protein crystallization experiment. 

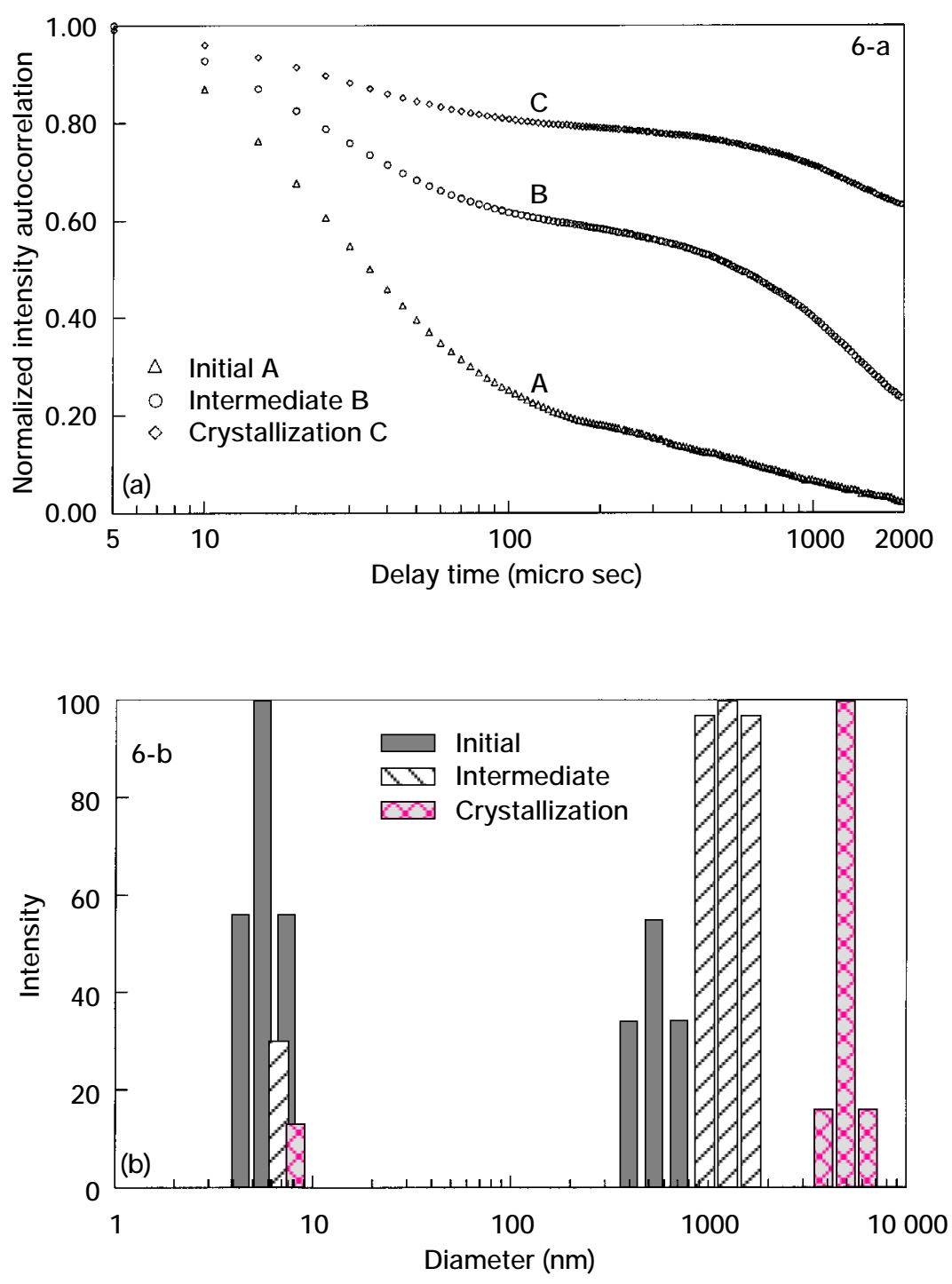

Figure 6.-Profiles representing various stages of crystal growth in the lysozyme system used in Figure 5. (a) Time correlation function (TCF). (b) Size distribution. 

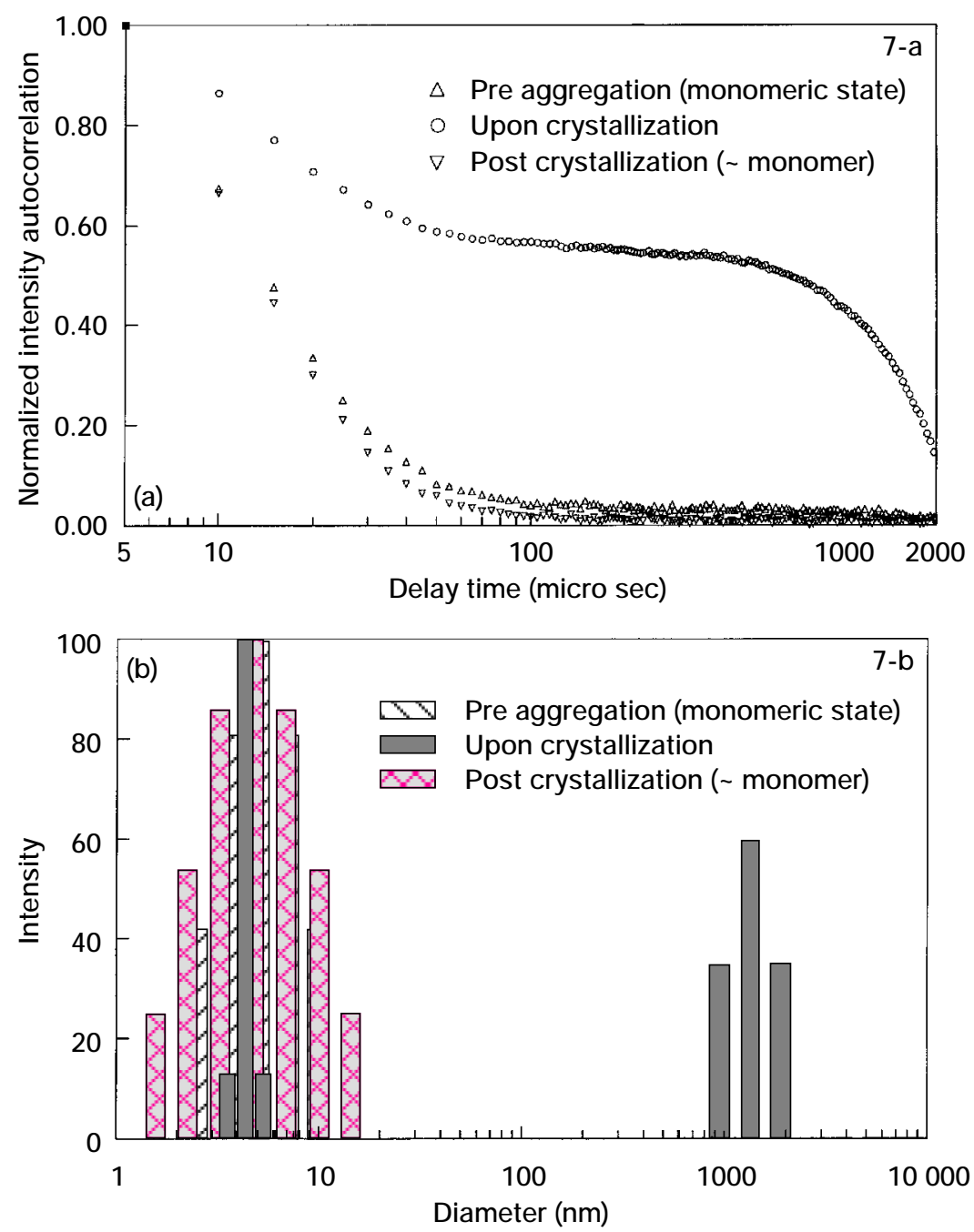

Figure 7.-Profiles representing various stages of crystal growth in a fast crystallizing thaumatin system. (a) TCF. (b) Size distribution.

\section{CONCLUSION}

We conclude that noninvasive, quantitative, fast, and reliable DLS measurements are now possible in hanging drop configurations suitable for ground-based and microgravity protein crystal growth facilities. The newly designed probe is capable of detecting the various particle sizes which may be associated with all of the various stages of protein crystal growth.

\section{ACKNOWLEDGMENTS}

The support of the Microgravity Science and Applications Division (Code UG) of the NASA headquarters is thankfully acknowledged. The probe was developed by R.R. Ansari and K.I. Suh at the NASA Lewis Research Center under grant number NCC 166-3/CWRU (Task Number 1566). The HDVDA system was developed by L.J. DeLucas and others and the DCVDS was developed by T.L. Bray and L.J. DeLucas at the UAB/CMC. The modified HDVDA for temperature-induced studies was developed by W.W. Wilson of MSU. KI. Suh would like to thank National Research Council (NRC) and the NASA Lewis Research Center of the award of a postdoctoral research fellowship. 


\section{REFERENCES}

1. Littke, W. and John, C., Science 225, p. 203, 1984.

2. Rosenblum, W.M., DeLucas, L.J., and Wilson, W.W., Protein Crystal Growth in Microgravity, in: Proc. NASA Laser Light Scattering Advanced Technology Development Workshop, NASA CP-10033, September, 1988, pp. 235-256.

3. Bray, T.L., Kim, L.J., Askew, R.P., Harrington, M.D., Rosenblum, W.M., Wilson, W.W., and DeLucas, L.J., New Crystallization Systems Envisioned for Microgravity Studies, J. Appl. Cryst., submitted.

4. Chu, B., Laser Light Scattering: Basic Principles and Practice (Academic Press, New York, 1991).

5. Ford, N.C., Theory and Practice of Correlation Spectroscopy, in: Measurements of Suspended Particles by Quasi-Elastic Light Scattering, Ed., Dahneke, B.E. (Wiley-Interscience, New York, 1983) pp. 31-78.

6. Ansari, R.R., Suh, K.I., DellaVecchia, M.A., and Dubin, S., Ophthalmic Diagnostics Using a New Dynamic Light Scattering Fiber Optic Probe, in: Proc. Medical Application, Conf. on Lasers in Ophthalmology III, September, 1995, Barcelona, Spain, to be published in Proc. SPIE-2632, No. 18.

7. Stock, R.S., and Ray, W.H., J. Polym. Sci.: Polym. Phys. Edition 23, p 1393, 1985.

8. Brown, R.G.W., Appl. Optics 26, 1987, p 4846; also Appl. Opt. 29, p. 4159, 1990.

9. Dhadwal, H.S., Method and Apparatus for Determining the Physical Properties of Materials Using Dynamic Light Scattering Techniques, U.S. patent No. 5,155,549, October 13, 1992.

10. Dhadwal, H.S., Ansari, R.R., and Meyer, W.V., Rev. Sci. Instrum 62 (12), p. 2963, 1991.

11. Casay, G.A., and Wilson, W.W., J. Crystal Growth 122, 1992, p. 95.

12 R.R. Ansari and K.I. Suh, Sizing of Colloidal Particles and Protein Molecules in a Hanging Fluid Drop, in: Proc. Biomedical Optoelectronics in Clinical Chemistry and Biotechnology, 12-16 September 1995, Barcelona, Spain, to be published in Proc. SPIE 2629, No. 23.

13. Malkin, A.J., and McPherson, A., Acta Cryst. D 50, 1994, p. 385. 
Public reporting burden for this collection of information is estimated to average 1 hour per response, including the time for reviewing instructions, searching existing data sources, gathering and maintaining the data needed, and completing and reviewing the collection of information. Send comments regarding this burden estimate or any other aspect of this collection of information, including suggestions for reducing this burden, to Washington Headquarters Services, Directorate for Information Operations and Reports, 1215 Jeffers
Davis Highway, Suite 1204, Arlington, VA 22202-4302, and to the Office of Management and Budget, Paperwork Reduction Project (0704-0188), Washington, DC 20503.
1. AGENCY USE ONLY (Leave blank)
2. REPORT DATE
November 1996
3. REPORT TYPE AND DATES COVERED
Technical Memorandum

\section{TITLE AND SUBTITLE}

A Fiber Optic Probe for Monitoring Protein Aggregation, Nucleation, and Crystallization

\section{AUTHOR(S)}

Rafat R. Ansari, Kwang I. Suh, Alireza Arabshahi, W. William Wilson, Terry L. Bray, and Lawrence J. DeLucas

\section{PERFORMING ORGANIZATION NAME(S) AND ADDRESS(ES)}

National Aeronautics and Space Administration

Lewis Research Center

Cleveland, Ohio 44135-3191

\section{SPONSORING/MONITORING AGENCY NAME(S) AND ADDRESS(ES)}

National Aeronautics and Space Administration

Washington, DC 20546-0001
5. FUNDING NUMBERS

WU-963-45-0A

NCC 166-3

8. PERFORMING ORGANIZATION REPORT NUMBER

E-10016

10. SPONSORING/MONITORING AGENCY REPORT NUMBER

NASA TM-107120

\section{SUPPLEMENTARY NOTES}

Prepared for the Sixth International Conference on Crystallization of Biological Macromolecules sponsored by the Hiroshima Prefecture, Hiroshima City, the Ministry of Education, Science, Sports and Culture, and the International Union of Chrystallography, Hiroshima, Japan, November 12-17, 1995. Rafat R. Ansari, NASA Lewis Research Center; Kwang I. Suh, National Research Council-NASA Research Associate at Lewis Research Center; Alireza Arabshahi and W. William Wilson, Mississippi State University, Department of Chemistry, P.O. Drawer CH, Mississippi State University, Mississippi 39762; Terry L. Bray and Lawrence J. DeLucas, University of Alabama at Birmingham, Center for Macromolecular Crystallography, Birmingham, Alabama 35294. Responsible person, Rafat R. Ansari, organization code 6712, (216) 433-5008; fax 216-977-7138; email ransari@lerc.nasa.gov.

12a. DISTRIBUTION/AVAILABILITY STATEMENT $\quad$ 12b. DISTRIBUTION CODE

Unclassified-Unlimited

Subject Categories 35, 51 and 74

This publication is available from the NASA Center for Aerospace Information, (301) 621-0390.

\section{ABSTRACT (Maximum 200 words)}

Protein crystals are experimentally grown in hanging drops in microgravity experiments on-board the Space Shuttle orbiter. The technique of dynamic light scattering (DLS) can be used to monitor crystal growth process in hanging droplets ( 30 (L)) in microgravity experiments, but elaborate instrumentation and optical alignment problems have made in-situ applications difficult. In this paper we demonstrate that such experiments are now feasible. We apply a newly developed fiber optic probe to various earth and space (microgravity) bound protein crystallization system configurations to test its capability. These include conventional batch (cuvette or capillary) systems, hanging drop method in a six-pack hanging drop vapor diffusion apparatus (HDVDA), a modified HDVDA for temperatureinduced nucleation and aggregation studies, and a newly envisioned dynamically controlled vapor diffusion system (DCVDS) configuration. Our compact system exploits the principles of DLS and offers a fast (within a few seconds) means of quantitatively and noninvasively monitoring the various growth stages of protein crystallization. In addition to DLS capability, the probe can also be used for performing single-angle static light scattering measurements. It utilizes extremely low levels of laser power ( few (W)) without a need of having any optical alignment and vibration isolation. The compact probe is also equipped with a miniaturized microscope for visualization of macroscopic protein crystals. This new optical diagnostic system opens up enormous opportunity for exploring new ways to grow good quality crystals suitable for x-ray crystallographic analysis and may help develop a concrete scientific basis for understanding the process of crystallization.

\begin{tabular}{|c|c|c|c|}
\hline \multicolumn{3}{|c|}{$\begin{array}{l}\text { 14. SUBJECT TERMS } \\
\text { Dynamic light scattering; Protein crystal growth; Droplet; Particle sizing; Microgravity }\end{array}$} & \multirow{2}{*}{\begin{tabular}{|} 
15. NUMBER OF PAGES \\
14 \\
16. PRICE CODE \\
A03 \\
\end{tabular}} \\
\hline Dynamic ingmi scalterng; & rotein crystal growtin, Dropiel, & articie sizing, ivicrogravity & \\
\hline $\begin{array}{l}\text { 17. SECURITY CLASSIFICATION } \\
\text { OF REPORT } \\
\text { Unclassified }\end{array}$ & $\begin{array}{l}\text { 18. SECURITY CLASSIFICATION } \\
\text { OF THIS PAGE } \\
\text { Unclassified }\end{array}$ & $\begin{array}{l}\text { 19. SECURITY CLASSIFICATION } \\
\text { OF ABSTRACT } \\
\text { Unclassified }\end{array}$ & 20. LIMITATION OF ABSTRACT \\
\hline NSN 7540-01-280-5500 & & & $\begin{array}{l}\text { Indard Form } 298 \text { (Rev. 2-89) } \\
\text { scribed by ANSI Std. Z39-18 } \\
-102\end{array}$ \\
\hline
\end{tabular}

